\title{
Arbeitsmarkteffekte von Industrieansiedlungen im ländlichen Raum
}

\section{Einleitung und Fragestellung}

Im Rahmen der bisher vorliegenden Beiträge zur Wirkunsanalyse der Industrieansiedlungspolitik dominieren rein unternehmensbezogene Betrachtungsansätze. Arbeitskräftebezogene Aspekte werden in der Regel vernachlässigt (1). Untersuchungen dieser Art sind insofern als einseitig zu bezeichnen, als aus regionalpolitischer Sicht höchst relevante Fragen zwangsläufig ausgeklammert werden (FREUND/ZABEL 1978, S. 65). Es bleibt insbesondere unklar, welche Menschen aus welchen Gründen die mit Hilfe von regionalpolitischen Maßnahmen geschaffenen Arbeitsplätze eingenommen haben. Werden die neuen Arbeitsplätze von der einheimischen Bevölkerung akzeptiert oder laufen die Abwanderungsprozesse mit ungeminderter Härte weiter? Sind die im Rahmen von Wirkungsanalysen häufig festgestellten, relativ ungünstigen Arbeitsplatzund Qualifikationsstrukturen neuangesiedelter Industriebetriebe in erster Linie angebotsbestimmt oder sind sie unter Umständen auch ein Zeichen dafür, da $\beta$ bestimmte Arbeitsmarktsegmente in peripheren Regionen besonders knapp und damit entsprechend schwierig zu rekrutieren sind (2)?

Vor diesem Hintergrund besteht das Ziel dieses Artikels darin, die Arbeitsmarkteffekte von Industrieansiedlungen im ländlichen Raum möglichst präzise nachzuzeichnen und zu erklären (3). Die für diese Zwecke notwendigen Daten wurden mittels einer Arbeitnehmerbefragung erhoben (4). Als Testgebiet diente die Region Sarneraatal, wo vorab in den fünfziger und sechziger Jahren eine recht erfolgreiche Industrieansiedlungspolitik betrieben wurde. In der Periode 1954 bis 1981 entstanden 23 neue Industriebetriebe mit insgesamt 1762 Arbeitsplätzen (1982). Damit entfallen in der Region Sarneraatal immerhin 75 Prozent aller industriellen Arbeitsplätze auf Neuansiedlungen (5).

\section{Frühere Tätigkeit der Arbeitskräfte}

Die Analyse der Tätigkeiten vor der Arbeitsaufnahme in einer der Neuansiedlungen läßt erkennen, da $\beta$ lediglich 65 Prozent der befragten Personen bereits früher berufstätig waren. Die übrigen 35 Prozent übten vor der jetzigen Anstellung noch keinen
Erwerb aus. In diesem Zusammenhang gilt es zu beachten, daß das Durchschnittsalter der Beschäftigten bei Neuansiedlungen relativ tief und deutlich unter dem Vergleichswert alteingesessener Industriebetriebe liegt. Über 70 Prozent der befragten Arbeitnehmer haben das vierzigste Altersjahr noch nicht überschritten. Am stärksten sind die Altersgruppen der 21-40jährigen vertreten.

Das geringe Durchschnittsalter der Beschäftigten bei den Neuansiedlungen ergibt sich aus dem Umstand, da $\beta$ jüngere Arbeitnehmer generell mobiler sind und insbesondere beim Eintritt ins Erwerbsleben eine hohe Mobilitätsbereitschaft aufweisen (6). Berufsanfänger sind am ehesten bereit, in einem neuen, d. h. noch unbekannten Betrieb und in einer unter Umständen früher noch nicht vertretenen Branche eine Arbeit anzunehmen. Bei den älteren Arbeitnehmern wirken hingegen die meist ausgeprägte Betriebstreue und die häufig konservative Grundhaltung mobilitätshemmend. Hinzu kommt, $\mathrm{da} ß$ die von den Neuansiedlungen nachgefragten Qualifikationen und Berufe von den älteren Arbeitnehmern nur beschränkt angeboten werden können.

Beinahe 70 Prozent der früher nicht Erwerbstätigen bekleiden heute eine Vollzeitstelle. Es handelt sich zum größten Teil um Schulabgänger, die in den regionalen Arbeitsmarkt integriert werden konnten. Rund 30 Prozent der befragten Arbeitskräfte haben einen Teilzeiterwerb aufgenommen. Bei dieser Gruppe dominieren Hausfrauen, die früher keinem Nebenerwerb nachgegangen sind. Völlig bedeutungslos war hingegen der Arbeitskräftetransfer aus der Landwirtschaft. Wie angesichts permanenter Vollbeschäftigung nicht anders zu erwarten war, blieb auch die Wiedereingliederung von Arbeitslosen auf Einzelfälle beschränkt.

Die Aufnahme der Erwerbstätigkeit in einer Neuansiedlung war nur bei rund einem Viertel aller Interaktionen mit einem interregionalen Wohnortwechsel verbunden. In diesem Fall kann nicht von einer Erhöhung der Erwerbsquote gesprochen werden. Hingegen wurden bei den übrigen drei Vierteln der Interaktionen brachliegende Potentiale aktiviert.

Christian Hanser, Dr., Walchestrasse 19, 8006 Zürich 
Die damit verbundene Erhöhung der Beschäftigungsintensität ist aus regionalpolitischer Sicht sehr erwünscht, da mit den jüngeren Arbeitnehmern ein räumlich besonders mobiles Arbeitsmarktsegment lokal gebunden werden konnte (LUTZ/SENGENBERGER 1974). Die Integration des beruflichen Nachwuchses ist in quantitativer Hinsicht sogar einer der bedeutendsten Arbeitsmarkteffekte, der im Rahmen der vorliegenden Untersuchung empirisch nachgewiesen werden konnte.

\section{Prozesse der beruflichen und sektoralen Mobilität}

\subsection{Berufliche Mobilitätsbereitschaft}

Um Konkurrenzierungen bereits ansässiger $\mathrm{Be}$ triebe zu vermeiden und aus stabilitätspolitischen Gründen ist die Industrieansiedlungspolitik in peripheren Regionen meistens auf eine möglichst weitgehende Branchendiversifizierung ausgerichtet. Die Realisierung eines Ansiedlungsprojektes führt deshalb im Rahmen einer solchen Strategie oft zur Schaffung von Arbeitsplätzen für Tätigkeiten, Berufe und Qualifikationen, die auf dem regionalen Arbeitsmarkt früher nicht oder nur in sehr kleiner Zahl nachgefragt wurden. Die Besetzung branchenfremder Arbeitsplätze ist nun allerdings nicht ganz einfach, da

- bei einem Standortbezug im ländlichen Raum in der Regel nicht mit markanten Zuwanderungsimpulsen gerechnet werden kann,

- auf den kleinen Arbeitsmärkten peripherer Regionen kurzfristig meist nur sehr geringe Arbeitskräftereserven mobilisiert werden können und

- die Integration des lokalen Nachwuchses bzw. die Bildung interner Arbeitsmärkte langsam vor sich geht und erst nach einigen Jahren zum gewünschten Erfolg führt.

Vor diesem Hintergrund wird ersichtlich, daß die Ergebnisse der Industrieansiedlungspolitik zwangsläufig unbefriedigend bleiben, wenn die berufliche Mobilitätsbereitschaft der einheimischen Arbeitskräfte eine gewisse Schwelle unterschreitet.

Tabelle 1 vermittelt für die berufliche Mobilitätsbereitschaft in der Region Sarneraatal ein recht günstiges Bild. Die Bereitschaft zu einem Berufs- undTätigkeitswechsel ist erstaunlich hoch und übertrifft die räumliche Mobilitätsbereitschaft bei weitem (vgl. Tabelle 4). Beinahe 60 Prozent aller Arbeitnehmer waren bei der Stellensuche auf keine bestimmte Tätigkeit festgelegt. Eine qualifikations- und geschlechtsspezifische Betrachtung zeigt, daß sich in sektorieller Hinsicht insbesondere die Frauen sowie die un- und angelernten Arbeitnehmer als außerordentlich flexibel erweisen. Die Männer und die qualifizierten Arbeitsmarktsegmente sind demgegenüber weit häufiger auf ein bestimmtesTätigkeitsfeld fixiert.

Besondere Erwähnung verdient der Sachverhalt, $\mathrm{da} \beta$ die berufliche Mobilitätsbereitschaft mit steigender Qualifikation nicht kontinuierlich abnimmt. Sehr große Unterschiede bestehen zwischen den Un- und Angelernten sowie dem Segment der Facharbeiter. Bedeutend geringere Differenzierungen ergeben sich hingegen zwischen dem Personal mit einfacher Berufslehre und den höher qualifizierten Arbeitnehmern. Hier sind bei der Stellenwahl beide Segmente ähnlich stark auf ein bestimmtes Berufsbzw. Tätigkeitsfeld fixiert. Damit wird die bekannte These der mit steigender Qualifikation immer geringer werdenden beruflichen Mobilitätsbereitschaft zwar grundsätzlich bestätigt (vgl. z. B. BUTT LER/GERLACH/LIEPMANN 1975, S. 70 oder HOFBAUER/ NAGEL 1973, S. 256), zugleich aber auch um einen wesentlichen Aspekt erweitert.

Tabelle 1 Berufliche Mobilitätsbereitschaft der Arbeitskräfte in neuen Industriebetrieben nach Qualifikation (Region Sarneraatal; Angaben in Spaltenprozent)

\begin{tabular}{|c|c|c|c|c|c|}
\hline $\begin{array}{ll}\text { Berufliche } & \text { Qualifikation } \\
\text { Mobilitätsbereitschaft } & \end{array}$ & $\begin{array}{l}\text { Un- und } \\
\text { angelernte } \\
\text { Arbeitskräfte }\end{array}$ & $\begin{array}{l}\text { Personal } \\
\text { mit } \\
\text { Berufslehre }\end{array}$ & $\begin{array}{l}\text { Höher } \\
\text { qualifiziertes } \\
\text { Personal }\end{array}$ & $\begin{array}{l}\text { Arbeitsk } \\
\text { absolut }\end{array}$ & in $\%$ \\
\hline $\begin{array}{l}\text { Ich wollte unbedingt auf meinem } \\
\text { Beruf arbeiten }\end{array}$ & 5 & 57 & 45 & 64 & 25 \\
\hline $\begin{array}{l}\text { ich wollte eine ganz bestimmte } \\
\text { Arbeit machen }\end{array}$ & 13 & 17 & 36 & 43 & 17 \\
\hline $\begin{array}{l}\text { ich habe mich im voraus auf keine } \\
\text { bestimmte Arbeit festgelegt }\end{array}$ & 82 & 26 & 19 & 147 & 58 \\
\hline Arbeitskräfte absolut & 149 & 74 & 31 & 254 & 100 \\
\hline
\end{tabular}


Tabelle 2 Sektorale Mobilität der Beschäftigten in neuen Industriebetrieben nach Branche und Geschlecht (Region Sarneraatal)

\begin{tabular}{|c|c|c|c|c|}
\hline $\begin{array}{l}\text { Tätigkeit in Branche } \\
\text { vor Stellenwechsel }\end{array}$ & $\begin{array}{l}\text { Anzahl Männer } \\
\text { absolut }\end{array}$ & $\begin{array}{l}\text { Anzahl Frauen } \\
\text { absolut }\end{array}$ & $\begin{array}{l}\text { Arbeits } \\
\text { absolut }\end{array}$ & $\begin{array}{l}\text { tota } \\
\text { in } \%\end{array}$ \\
\hline $\begin{array}{l}\text { Landwirtschaft/Steinbrüche, Gruben } \\
\text { Industrie/produzierendes Gewerbe } \\
\text { davon: } 20 / 21 \text { Nahrungsmittel } \\
\text { 23/24 Textil/Bekleidung } \\
25 \text { Holz und Kork } \\
28 \text { Graphik } \\
30 \text { Kunststoff } \\
33 \text { Steine und Erden } \\
34 \text { Metall } \\
35 \text { Maschinen } \\
\text { - Übrige Industrie } \\
\text { Baugewerbe } \\
\text { Private Dienstleistungen } \\
\text { Öffentliche Verwaltungen }\end{array}$ & $\left.\begin{array}{r}2 \\
86(78 \%) \\
3 \\
1 \\
12 \\
2 \\
1 \\
- \\
13 \\
21 \\
33 \\
5 \\
12 \\
5\end{array}\right\}(15 \%)$ & $\left.\begin{array}{l}1 \\
27(44 \%) \\
2 \\
9 \\
1 \\
1 \\
2 \\
1 \\
1 \\
4 \\
6 \\
2 \\
29 \\
1\end{array}\right\}(49 \%)$ & $\left.\begin{array}{r}3 \\
113 \\
5 \\
10 \\
13 \\
3 \\
3 \\
1 \\
14 \\
25 \\
39 \\
7 \\
41 \\
6\end{array}\right\}$ & $\begin{array}{r}2 \\
66\end{array}$ \\
\hline Arbeitskräfte absolut & 110 & 60 & 170 & 100 \\
\hline
\end{tabular}

Keine Antwort: 1

\subsection{Sektorale Mobilität}

Die Befunde zur sektoralen Mobilität (7) zeigen, $\mathrm{da} ß$ der Stellenantritt in einem der neuen Industriebetriebe bei 81 Prozent der bereits früher erwerbstätigen Arbeitnehmer mit einem Branchenwechsel verbunden war. Dieser rege Branchenwechsel muß mit den vorangehend präsentierten Ergebnissen einer deutlich geringeren beruflichen Mobilitätsbereitschaft nicht unbedingt im Widerspruch stehen. Dieser löst sich nämlich auf, wenn man in Erwägung zieht, daß verschiedene Berufe und Tätigkeiten an keine bestimmte Branche gebunden sind. Die Wahl des Arbeitsplatzes richtet sich primär nach Berufs- und Tätigkeitsfeldern und erst sekundär nach der Branche.

Eine detailliertere Betrachtung dieses Sachverhaltes zeigt (vgl. Tabelle 2), daß zwei Drittel aller Arbeitskräfte bereits früher im sekundären Sektor gearbeitet haben. Rund ein Drittel der Belegschaft der Neuansiedlungen ging vor dem Stellenwechsel einer Tätigkeit im Dienstleistungssektor nach. Der Arbeitskräftetransfer aus der Landwirtschaft ist hingegen - wie bereits früher festgestellt - äußerst bescheiden. Offensichtlich fällt auch in der Region Sarneraatal eine landwirtschaftliche Betriebsaufgabe meistens mit der Erwerbsaufgabe des $\mathrm{Be}$ triebsleiters zusammen, so daß aus diesem schrumpfenden Sektor eigentlich gar nie größere Arbeitskräftereserven freigestellt werden.

\subsection{Motive für den Arbeitsplatzwechsel}

Im vorliegenden Kapitel soll aus der subjektiven Sicht der Befragten festgestellt werden, welche
Aspekte bei derWahl des Arbeitsplatzes als besonders wichtig erachtet wurden. Tabelle 3 läßt in bezug auf diese Frage erkennen, da $\beta$ die von den Neuansiedlungen geschaffenen Arbeitsplätze in erster Linie deshalb angenommen wurden, weil sie dauerhaft erschienen, eine abwechslungsreiche Tätigkeit versprachen und gut erreichbar waren. Weniger wichtig waren demgegenüber Aspekte wie eine gute Bezahlung, Aufstiegsmöglichkeiten oder flexible Arbeitszeiten (8).

Mit Bezug auf die qualifikationsspezifische Betrachtung ist festzuhalten, da $B$

- das allgemeine Anspruchsniveau mit steigender beruflicher Position höher wird,

- ein dauerhafter und gut erreichbarer Arbeitsplatz vor allem bei den un- und angelernten Arbeitskräften besonders stark gewichtet wird,

- bei den besser ausgebildeten Segmenten die Art der Tätigkeit (Vielseitigkeit, Abwechslung etc.) von hervorragender Bedeutung ist,

- den Löhnen auf jedem Niveau höchstens eine mittlere Bedeutung beigemessen wird (9) und

- man den Aufstiegsmöglichkeiten bzw. dem mit dem Stellenwechsel vollzogenen Aufstieg mit steigender Qualifikation immer mehr Beachtung schenkt (10).

Aus diesen Befunden ist der wichtige Schluß zu ziehen, da $\beta$ dezentralisierte Ansiedlungsstrategien den Bedürfnissen der einheimischen Arbeitskräfte peripherer Regionen besonders gut entsprechen, wenn in erster Linie Arbeitsplätze für Un- und Angelernte und für Frauen geschaffen werden. Diese beiden Segmente zeigen eine eindeutige Vorliebe für Arbeitsplätze im engsten Wohnumfeld und sind 
Tabelle 3 Motive für Stellenwechsel nach Qualifikation

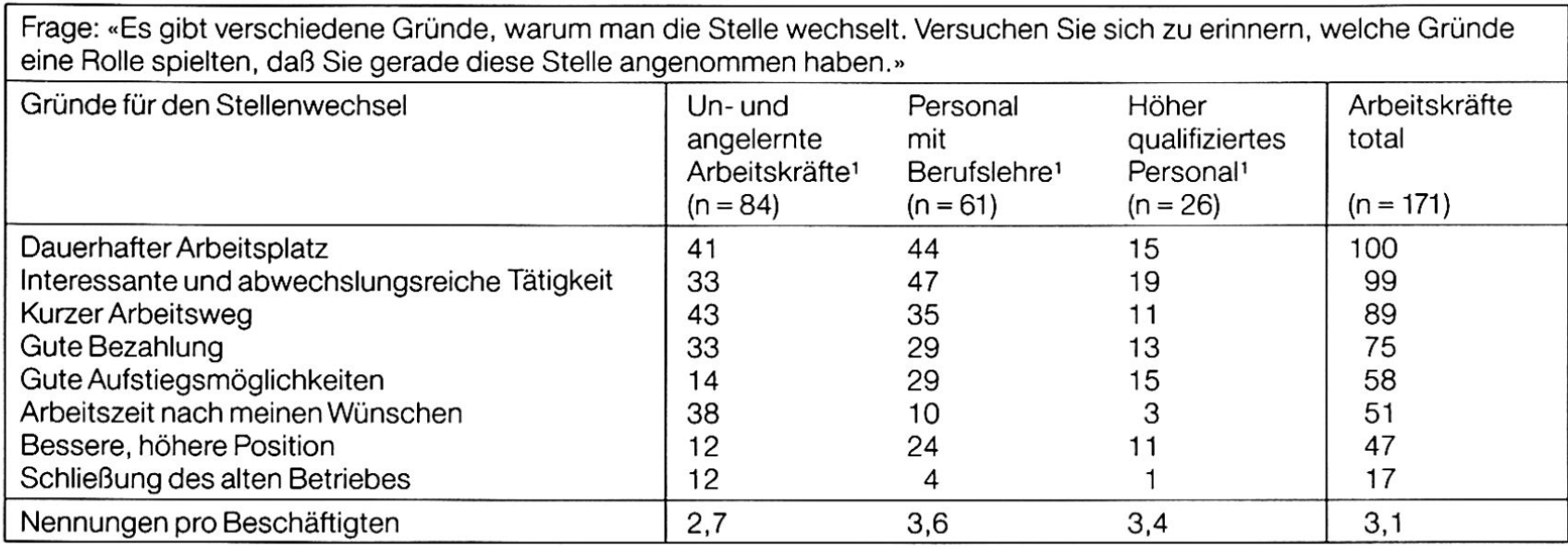

1 Anzahl Nennungen sehr wichtig;

Mehrfachnennungen möglich

offenbar durchaus bereit, für den Vorteil der guten Erreichbarkeit in anderen Bereichen gewisse Abstriche in Kauf zu nehmen (Art der Tätigkeit, Löhne, Aufstiegschancen). Bei den qualifizierteren Arbeitsmarktsegmenten verlieren die räumlichen Muster der Industrialisierung an Bedeutung. Wichtiger werden hier all diejenigen Faktoren, die für eine erfolgsversprechende berufliche Karriere bedeutungsvoll sind.

\section{Prozesse der regionalen Mobilität}

\subsection{Räumliche Wanderungsbereitschaft}

«Die Analyse derWanderungsbereitschaft ist für die regionalpolitische Bewertung von Industrieansiedlungen insofern relevant, als sich ableiten läßt, ob die Ansiedler stark regionsverhaftete Menschen beschäftigen, die nicht nur (. . .) in der Vergangenheit sehr wenig interregional gewandert sind, sondern auch in Zukunft nur im äußersten Falle ihre Heimatregion verlassen werden oder ob die Beschäftigten, die sich mit dem Arbeitsplatzwechsel als berufsbezogen mobil ausgewiesen haben, auch regional trotz der bislang geringen Wanderungen potentiell mobil sind und nur durch die Ansiedler in der Region gehalten werden konnten" (FREUND/ZABEL 1978, S. 69). Im Rahmen der vorliegenden Untersuchung ermöglicht die Erforschung der räumlichen Wanderungsbereitschaft zudem eine Überprüfung der arbeitnehmerbezogenen Prämissen des derzeitigen Konzeptes der Berggebietsförderung. Es stellt sich in diesem Zusammenhang vorab die Frage, ob das gegenwärtige Förderkonzept der aktiven Sanierung den Wohnpräferenzen der Betroffenen überhaupt gerecht wird. Drängen nicht große Bevölkerungsan- teile peripherer Regionen in die Ballungsgebiete, weil sie dort bessere Erwerbsmöglichkeiten, Wohnund Freizeitwerte aufzufinden hoffen (11)?

Die empirischen Befunde zur räumlichen Wanderungsbereitschaft lassen keine Zweifel offen, da das Förderkonzept der aktiven Sanierung den Wohnpräferenzen großer Bevölkerungskreise peripherer Regionen in sinnvoller Weise Rechnung trägt (vgl. Tabelle 4): 74 Prozent der Befragten wollten die Arbeitsmarktregion Sarneraatal bei der Stellenwahl auf keinen Fall verlassen; lediglich 26 Prozent hätten sich vorstellen können, auch in einer ganz anderen Gegend zu arbeiten.

Deutlich zeigt sich auch in diesem Zusammenhang die schichtspezifische Prägung der Mobilitätsmuster: Mit steigender Qualifikation nimmt die Ortsgebundenheit der Arbeitnehmer eindeutig ab (12). Ähnlich wie bei der beruflichen Mobilitätsbereitschaft zeichnet sich auch hier keine lineare Funktion ab. Die Arbeitskräfte mit Berufslehre unterscheiden sich hinsichtlich räumlicher Wanderungsbereitschaft vom Segment der Un- und Angelernten nur unwesentlich. Die großen Abweichungen liegen hier zwischen den Verhaltensweisen der Facharbeiter und den höher qualifizierten Arbeitnehmern. Damit findet auch die allgemein anerkannte These der mit steigendem beruflichem Status abnehmenden räumlichen Bindung der Arbeitnehmer eine wesentliche Präzisierung.

Zur Charakterisierung der Arbeitsmarktverhältnisse in der Region Sarneraatal ist es nun wichtig, die bisher getrennt betrachteten Befunde zur räumlichen und beruflichen Mobilitätsbereitschaft einer kombinierten Analyse zu unterziehen. Dabei wird deutlich, daß

- immerhin 30 Prozent aller befragten Arbeitnehmer sowohl in beruflicher als auch in regionaler Hinsicht als immobil zu betrachten sind; 
Tabelle 4 Räumliche Mobilitätsbereitschaft der Arbeitskräfte in neuen Industriebetrieben nach Qualifikation (Region Sarneraatal; Angaben in Spaltenprozent)

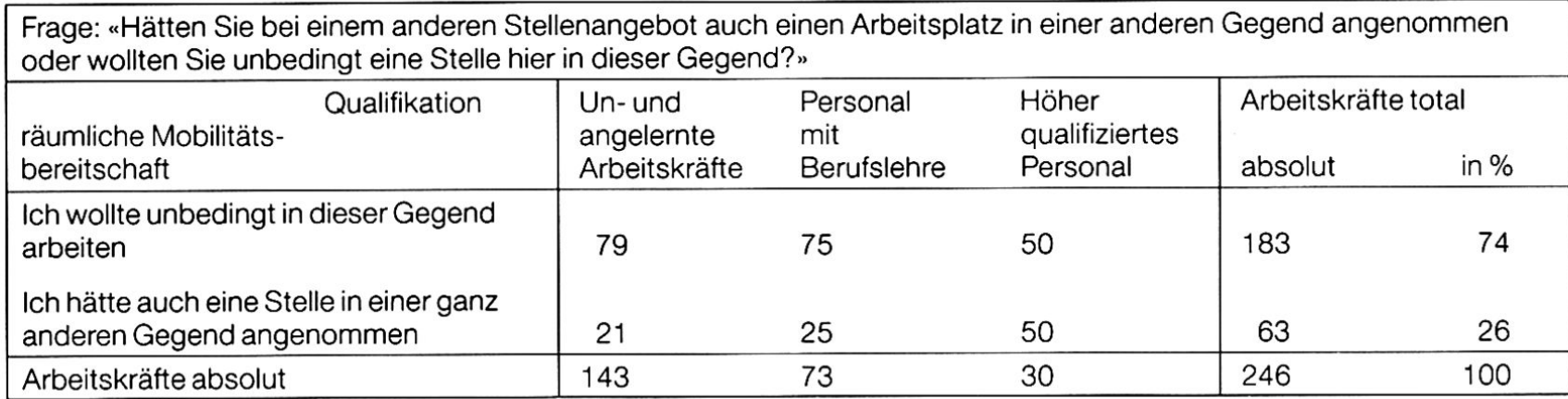

Keine Antwort: 18

Quelle: Eigene Erhebungen

- der Anteil der beruflich aber nicht regional mobilen Arbeitskräfte deutlich größer ist (44\%) als der Anteil der Arbeitskräfte, die sich als regional aber nicht beruflich mobil bezeichen (13\%);

- lediglich 13 Prozent der bei den Neuansiedlungen beschäftigten Arbeitnehmer in jeder Hinsicht mobil waren.

In diesen Ergebnissen spiegeln sich zunächst die Befunde, daß die (vielen) Un- und Angelernten eher zu sektoraler Mobilität bereit sind und die (wenigen) höher qualifizierten Arbeitnehmer vor allem eine große räumliche Wanderungsbereitschaft an den Tag legen. Die qualifikationsspezifische Differenzierung dieser Egebnisse führt aber auch zum überraschenden und bisher wenig bekannten Ergebnis, daß die gelernten Fachkräfte die insgesamt geringste Mobilitätsbereitschaft aufweisen. Gelernte Berufsleute sind in beruflicher Hinsicht beinahe so stark auf eine bestimmte Tätigkeit fixiert wie die höher qualifizierten Arbeitnehmer und in räumlicher Hinsicht beinahe so stark gebunden wie die Un- und Angelernten. Aus dieser Optik wird die geringe Mobilität der gelernten Facharbeiter zu einem der Kernprobleme kleiner und peripherer Arbeitsmärkte (13). Der in ländlichen Regionen sehr häufig beklagte, chronische Facharbeitermangel kann auf diese Weise einleuchtend erklärt werden.

\subsection{Intra- und interregionale Wanderungen}

Im Zentrum dieses Kapitels steht die Analyse der durch die industriellen Betriebsansiedlungen in der Region Sarneraatal ausgelösten räumlichen Wanderungen von Arbeitskräften und deren Familien. Die in diesem Zusammenhang wesentlichen Befunde zeigen, daß

- der Stellenantritt bei 42 Prozent aller Arbeitnehmer keinen Wohnsitzwechsel erfordert,

- 22 Prozent der Befragten einen intraregionalen Wohnsitzwechsel realisierten,
- 36 Prozent der Arbeitnehmer als interregionale Zuzüger zu bezeichnen sind und

- die Zahl der Zuwanderer aus dem Ausland - diese werden in der Folge ebenfalls zur Gruppe der interre gionalen Zuzüger gezählt - äußerst bescheiden ist (12 Personen) (14).

Diese Befunde sind mit den Ergebnissen früherer Analyseteilschritte kompatibel. Sie sind ein weiteres Indiz dafür, daß auf dem Arbeitsmarkt die kleinräumigen Anpassungsprozesse überwiegen. Die geringen Interaktionsdistanzen lassen sich angebotsseitig dadurch erklären, daß die im Zuge der Industrialisierung geschaffenen Arbeitsplätze den Ansprüchen des regionalen Arbeitsmarktes offenbar auch in qualitativer Hinsicht recht gut entsprechen. Nachfrageseitig ist wesentlich, daß die einheimischen Arbeitskräfte eine sehr geringe räumliche Mobilitätsbereitschaft aufweisen und allfällige Arbeitsmarktungleichgewichte eher durch sektorale bzw. berufliche Mobilität zu überwinden trachten.

\subsection{Wanderungsmotive und Wohnattraktivität}

Die Analyse der Wanderungsmotive soll zeigen, inwieweit die im letzten Kapitel dargestellten Prozesse der räumlichen Mobilität mit dem Stellenwechsel in ursächlichem Zusammenhang stehen. Wurden die beobachteten inter- und intraregionalen Wanderungen durch die bei den Neuansiedlungen geschaffenen Arbeitsplätze ausgelöst oder spielten andere Umzugsgründe wie $z$. B. das Vorfinden einer geeigneten Wohnung oder die gute Umweltqualität der Region Sarneraatal ebenfalls eine wichtige Rolle? Von erheblicher Bedeutung ist dabei aber nicht zuletzt auch die Frage nach dem Stellenwert der haushaltorientierten Infrastruktur im Wohnortentscheid mobiler Arbeitnehmer. Ist es tatsächlich möglich, das Wanderungsverhalten von Arbeitskräften mit Hilfe von infrastrukturellen Maßnahmen zielgerecht zu beeinflussen, wie dies im Rahmen re- 
gionalpolitischer Strategiediskussionen immer wieder unterstellt wird (15)?

Die Auswertung der Befragungsergebnisse läßt erkennen, daß die interregionalen Wanderungen in erster Linie aus beruflichen Gründen erfolgten. Bei knapp 60 Prozent aller außerregionalen Zuzüger spielten Arbeitsplatzmotive eine wesentliche Rolle (16). Bereits deutlich weniger wichtig sind die an zweiter Stelle zu erwähnenden wohnungsbezogenen Umzugsgründe. Ledigleich 20 Prozent aller interregionalen Wanderungen lassen sich durch einen Liegenschaftserwerb oder die Möglichkeit der Miete einer passenden Wohnung erklären.

Überraschenderweise folgen bereits in dritter Position umweltbezogene Wanderungsmotive: 16 Prozent aller interregionalen Zuwanderer geben an, vor allem wegen der guten Umweltqualität in der Region Sarneraatal Wohnsitz bezogen zu haben. Persönliche, infrastrukturelle oder freizeitbezogene Wanderungsmotive sind bei großräumigen Interaktionen indessen offensichtlich von untergeordneter Bedeutung.

Demgegenüber steht bei interregionalen Wanderungen eindeutig die Suche nach geeignetem Wohnraum im Vordergrund. 49 Prozent der interregionalen Interaktionen erfolgten, weil in der Zuzugsgemeinde eine passende Wohnung bezogen oder ein eigenes Haus gekauft werden konnte. Aus Arbeitsplatzgründen wird innerhalb der Region Sarneraatal eindeutig weniger häufig gewandert (39\%). Umwelt-, Infrastruktur- oder persönliche Faktoren sind auch auf dieser Betrachtungsebene nahezu bedeutungslos.

Die Resultate dieses Analyseschrittes decken sich weitgehend mit den Erkenntnissen der neueren Wanderungsforschung (vgl. z. B. BASSAND/BRULHARDT/HAINARD/SCHULER 1985; SCHULER 1984; BASSAND/BRULHARDT 1980). Stellvertretend sei etwa auf die Charakterisierung interregionaler Migrationsprozesse bei OECHSLIN (1981, S. 125) hingewiesen, die durch vorliegende Ergebnisse in allen wesentlichen Punkten bestätigt wird: «Primärer Antrieb für eine interregionale Wanderung ist eine unbefriedigende wirtschaftliche oder berufliche Situation oder aber die Attrahierungskraft einer andern sich bietenden Stelle. Die Entscheidung, ob überhaupt gewandert wird, fällt somit primär aufgrund wirtschaftlicher Überlegungen. Für die Richtung der Wanderung sind zusätzlich die allgemeinen Wohnortwünsche bzw. die regionalen Präferenzen entscheidend. Wer einen neuen Arbeitsplatz sucht, wird nur in solchen Gebieten nach einer Anstellung Umschau halten, die seiner regionalen Präferenz entsprechen. Andere Gebiete jedoch, die den regionalen Präferenzen nicht entsprechen, werden nur dann in Betracht gezogen, wenn die Arbeitsmarktverhältnisse keine beliebigen Wahlmöglichkeiten offenlassen oder wenn man von einer offenen Stelle erfährt, die ganz besonders attraktiv erscheint. So- mit haben neben den beruflich-wirtschaftlichen Einflußfaktoren auch die allgemeinen Wohnortwünsche eine große Bedeutung für interregionale Wanderungen».

Übereinstimmungen ergeben sich auch in bezug auf die Bewertung der Infrastruktur. Ein Blick auf die Literatur zeigt, daß die Infrastruktur bei sämtlichen vergleichbaren Migrationsanalysen von nachrangiger Bedeutung ist. Sie bleibt zwar bei einem Wohnungswechsel nicht unbeachtet, sie hat indessen weder bei groß- noch bei kleinräumigen Wanderungen entscheidungsprägenden Charakter. Gestützt auf verhaltenstheoretische Überlegungen hält OECHSLIN (1981, S. 143) fest, daß «einzelne Infrastruktureinrichtungen für sich allein keine genügende Attrahierungskraft für interregionale Wanderungen haben können».

Tabelle 5 läßt nun allerdings erkennen, daß die Wohnqualität einer Region - trotz der geringen $\mathrm{Be}$ deutung der Infrastruktur als Wanderungsmotiv - in erheblichem Masse durch infrastrukturelle Faktoren geprägt wird. Eine gute medizinische Versorgung, vielfältige Einkaufsmöglichkeiten und ein breites Bildungsangebot sind Faktoren, die für die Mehrheit der Bevölkerung von größter Bedeutung sind. Die Skala der wichtigsten Wohn- und Freizeiteigenschaften zeigt, daß den genannten Infrastrukturfaktoren lediglich noch die Umweltqualität übergeordnet wird. Als weniger wichtig werden in der Region Sarneraatal hingegen die sportlichen und kulturellen Betätigungsmöglichkeiten eingestuft (17).

Aus den Ergebnissen dieses Analyseschrittes ergeben sich in zweierlei Hinsicht einige wesentliche Schlußfolgerungen. In bezug auf die Frage der Arbeitsmarktverflechtungen scheint wichtig, da $\beta$

- knapp 60 Prozent aller interregionalen Wanderungen mit der Annahme einer Stelle bei einem der neuen Industriebetriebe in direktem Zusammenhang stehen (diese Akteure wären ohne die betreffenden Arbeitsplätze mit großer Wahrscheinlichkeit nicht in die Region Sarneraatal gezogen);

- rund 40 Prozent der interregionalen Interaktionen vorwiegend auf Wohnungs- und Umweltgründe zurückzuführen sind (die Akteure dieser Gruppe hätten den Arbeitsplatz bei einer der Neuansiedlungen mehrheitlich auch ohne Wohnsitzwechsel angenommen; das Auseinanderfallen von Wohn- und Arbeitsort hätte allerdings längere Pendlerwege erfordert);

- die interregionalen Wanderungen nur zu knapp 40 Prozent als arbeitsplatzbedingt anzusehen sind;

- der größere Teil der interregionalen Wanderungen mit der Wahl des Arbeitsplatzes in keinerlei Verbindung steht.

Die Befunde hinsichtlich der Bedeutung der Infrastruktur bei der Wohnortwahl lassen sich in Thesenform wie folgt zusammenfassen: 
Tabelle 5 Bewertung der Wohnfaktoren durch die Beschäftigten in neuen Industriebetrieben nach Qualifikation (Region Sarneraatal)

\begin{tabular}{|c|c|c|c|c|}
\hline \multicolumn{5}{|c|}{$\begin{array}{l}\text { Frage: “Jeder Mensch hat seine bestimmten Vorstellungen von einem idealen Wohnort. Kreuzen Sie bitte in der nach- } \\
\text { folgenden Liste bei jeder der aufgezählten Wohn- und Freizeiteigenschaften an, ob sie für Sie persönlich sehr wichtig. } \\
\text { weniger wichtig oder unwichtig ist. Es spielt dabei keine Rolle, ob diese Eigenschaft an Ihrem Wohnort vorhanden ist } \\
\text { oder nicht." }\end{array}$} \\
\hline \multirow[t]{2}{*}{ Wohnortfaktoren } & \multicolumn{4}{|c|}{$\begin{array}{l}\text { Prozentsatz der Personen, welche diesen Faktor als "Sehr wichtig" } \\
\text { bezeichnen }{ }^{1}\end{array}$} \\
\hline & $\begin{array}{l}\text { Un- und } \\
\text { angelernte } \\
\text { Arbeitskräfte } \\
(n=156)\end{array}$ & $\begin{array}{l}\text { Personal } \\
\text { mit } \\
\text { Berufslehre } \\
(n=76)\end{array}$ & $\begin{array}{l}\text { Höher } \\
\text { qualifiziertes } \\
\text { Personal } \\
(n=32)\end{array}$ & $\begin{array}{l}\text { Arbeitskräfte total } \\
(n=264)\end{array}$ \\
\hline $\begin{array}{l}\text { Reizvolle landschaftliche Umgebung und } \\
\text { gute Umweltqualität } \\
\text { Gute ärztliche Versorgung } \\
\text { Gute Einkaufsmöglichkeiten für } \\
\text { Lebensmittel } \\
\text { Gute Straßenverbindungen } \\
\text { Nähe zu Oberstufenschulen (Sekundar-, } \\
\text { Real-, Bezirksschule) } \\
\text { Gute Bahn- und Busverbindungen } \\
\text { Gute Sportmöglichkeiten } \\
\text { Nähe zu einer Berufsschule } \\
\text { Weiterbildungsmöglichkeiten für } \\
\text { Erwachsene } \\
\text { Nähe zu Einkaufsmöglichkeiten für Kleider, } \\
\text { Bücher, Möbel usw. } \\
\text { Nähe zu einer Mittelschule } \\
\text { (Gymnasium, Seminar usw.) } \\
\text { Reiches Vereins- und Kulturleben (Theater- } \\
\text { veranstaltungen, Gesangsverein usw.) } \\
\text { Gute Gaststätten und Restaurants }\end{array}$ & $\begin{array}{l}78 \% \\
67 \% \\
\\
62 \% \\
57 \% \\
\\
46 \% \\
42 \% \\
35 \% \\
37 \% \\
30 \% \\
32 \% \\
17 \% \\
\\
\\
\\
19 \% \\
17 \% \\
\end{array}$ & $\begin{array}{l}97 \% \\
71 \% \\
\\
55 \% \\
62 \% \\
\\
42 \% \\
42 \% \\
49 \% \\
37 \% \\
\\
41 \% \\
\\
25 \% \\
\\
\\
22 \% \\
\\
25 \% \\
12 \% \\
\end{array}$ & $\begin{array}{l}94 \% \\
17 \% \\
\\
47 \% \\
28 \% \\
\\
56 \% \\
22 \% \\
31 \% \\
34 \% \\
\\
56 \% \\
\\
16 \% \\
\\
41 \% \\
\\
\\
22 \% \\
13 \% \\
\end{array}$ & $\begin{array}{l}86 \% \\
66 \% \\
58 \% \\
55 \% \\
\\
43 \% \\
39 \% \\
38 \% \\
36 \% \\
36 \% \\
28 \% \\
22 \% \\
21 \% \\
15 \% \\
\end{array}$ \\
\hline Nennungen pro Beschäftigten & $5,3 \%$ & $5,8 \%$ & $5,1 \%$ & 5,4 \\
\hline
\end{tabular}

1 Mehrfachnennungen möglich

- Das Wanderungsverhalten der Arbeitskräfte wird in erster Linie von Arbeitsplatz- und Wohnungsmotiven geleitet. Infrastrukturleistungen werden sowohl bei großräumiger als auch bei kleinräumiger Migration nur in Einzelfällen entscheidungswirksam.

- Verschiedene Infrastrukturleistungen (medizinische und schulische Versorgung; Einkaufsmöglichkeiten) sind nun allerdings als Wohnortfaktor von so großer Bedeutung, daß Defizite über eine längere Periode kaum akzeptiert werden dürften.

- Die Formel "notwendig, aber nicht hinreichend" (FREY 1979, S. 68) hat somit auch im Bereich der haushaltorientierten Infrastruktur Gültigkeit. Auch im vorliegenden Zusammenhang ist davon auszugehen, daß vorhandene Infrastrukturangebote nicht attrahierend, Infrastrukturmängel hingegen zuzugshemmend und abwanderungsfördernd wirken.

\section{Zusammenfassung}

(1) Die bei den neuen Industriebetrieben in der Region Sarneraatal beschäftigten Arbeitnehmer wei- sen ein relativ geringes Durchschnittsalter auf. Die Belegschaften sind in der Regel jünger als in alten Industriebetrieben.

(2) Ein eher ungünstiges Bild ergibt sich hingegen in bezug auf die Ausbildung der Arbeitskräfte: Gemessen an den formalen beruflichen und schulischen Ausbildungskriterien dominiert ganz eindeutig das Segment der Un- und Angelernten.

(3) Ein synoptischer Überblick über die Anpassungsprozesse auf dem regionalen Arbeitsmarkt (vgl. Tabelle 6) läßt erkennen, daß 35 Prozent der Belegschaft der Neuansiedlungen vor dem Stellenantritt nicht berufstätig waren. Der Effekt der Integration früher erwerbsloser Arbeitnehmer in den Produktionsproze $B$ ist umfangmä $\beta$ ig bedeutender als alle andern Teilwirkungssequenzen. Er trägt nicht unwesentlich zur Verbesserung der Einkommensverhältnisse bei (Erwerbstätigkeit der Frauen) und hilft, einen Teil der beruflichen Nachwuchstätigkeit lokal zu binden. Es ergeben sich mithin auch Impulse zur Erhöhung der Erwerbsquote.

(4) 24 Prozent der befragten Arbeitskräfte waren vor dem Stellenwechsel bei einem andern regionalen Betrieb beschäftigt. In den meisten Fällen 
Tabelle 6 Die durch die Industrieansiedlungspolitik ausgelösten Arbeitsmarktprozesse im Überblick (nach Qualifikation)

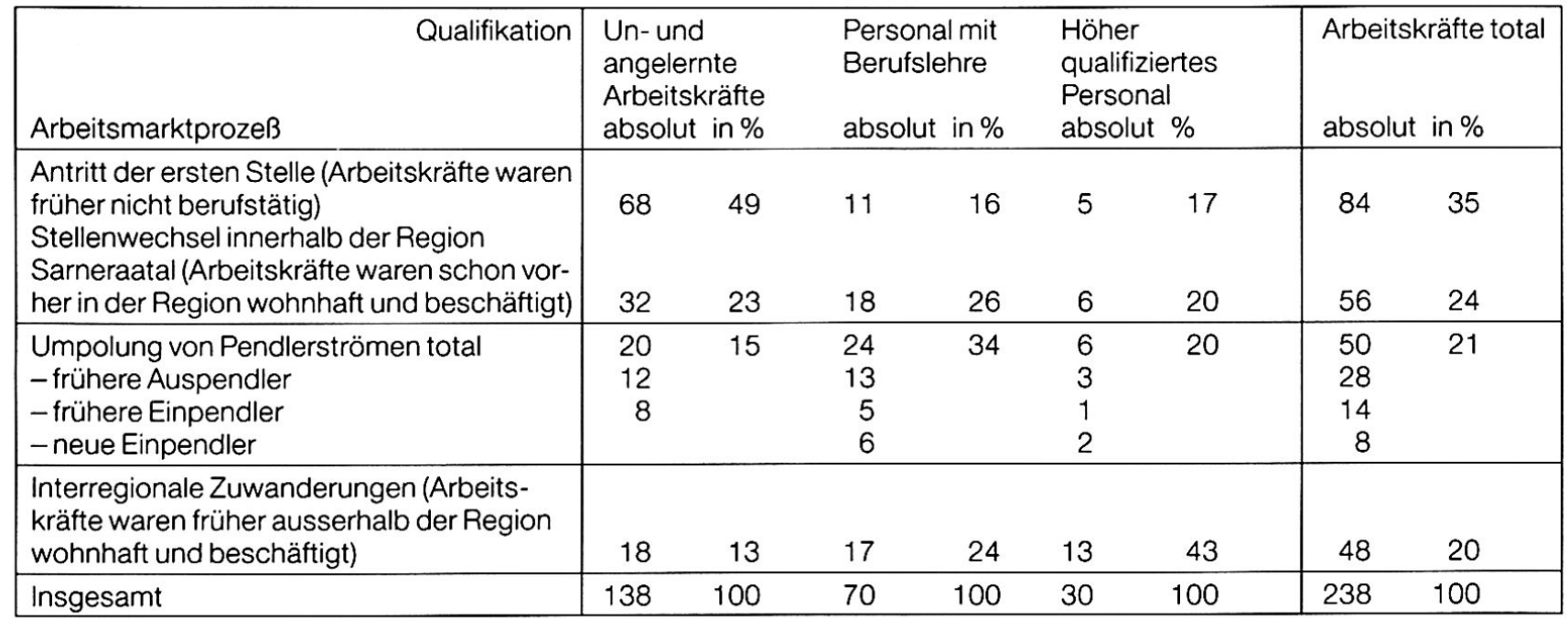

keine Antwort: 26

Quelle: Eigene Erhebungen

konnte mit dem Stellenwechsel eine Verbesserung der Einkommensverhältnisse realisiert werden. In der Regel dominieren in diesem Zusammenhang die Prozesse der sektoralen Mobilität.

(5) Schließlich bewirkten die Arbeitsplätze der Neuansiedlungen auch eine gewisse interregionale $\mathrm{Zu}-$ wanderung. Dieser Effekt fällt in quantitativer Hinsicht am schwächsten aus, ohne allerdings gegenüber den vorangehend diskutierten Teilwirkungen deutlich abzufallen.

\section{Anmerkungen}

1 Unter den wenigen Ausnahmen befinden sich zum Beispiel die Arbeiten von NUPPNAU (1974) oder SPEHL/TOEPFER/ TOEPFER (1975).

2 KELLER (1983, S. 144) weist in diesem Zusammenhang zu Recht darauf hin, "daß die Nachfrage nicht einseitig durch das Angebot auf dem Arbeitsmarkt bestimmt wird, sondern daß sich das Angebot in gewissen Grenzen der Nachfrage anpaßt".

3 Die in diesem Zusammenhang relevanten theoretischen Überlegungen sind ausführlich dargestellt bei HANSER (1986, S. 64 ff.).

${ }^{4}$ Das Sample umfaßt insgesamt 713 Arbeitnehmer bei neuen Industriebetrieben; die Rücklaufquote beträgt $37 \%$ (vgl. HANSER 1986, S. 76/77).

5 Eine ausführliche Erfolgsbeurteilung der Industrieansiedlungspolitik in der Region Sarneraatal liegt vor bei HANSER (1982, S. 45 f.).

6 GATZWEILER (1975, S. 49) weist nach, daß die Migrationsprozesse im ländlichen Raum durch eine starke altersselektive Schichtung gekennzeichnet sind. Als besonders mobil erweisen sich die 16-20jährigen (Bildungswanderer) sowie die 31-34jährigen (qualifizierte Arbeitsplatzwanderer). Nach dem 35. Altersjahr nimmt die Arbeitsplatzmobilität stark ab. Die Wanderungen erfolgen nach diesem Zeitpunkt in erster Linie zur Verbesserung des indivduellen Wohnumfeldes.

7 In Anlehnung an FREUND/ZABEL (1978, S. 66 f.) werden im Rahmen der vorliegenden Untersuchung die Begriffe der sektoralen Mobilität und der branchenbezogenen Mobilität synonym verwendet. Ein Arbeitnehmer ist demnach sektoral mobil, wenn er gleichzeitig mit dem Stellenwechsel auch eine Tätigkeit in einer andern Branche aufnimmt. Mit Blick auf eine zusätzliche Präzisierung ist an dieser Stelle festzuhalten, daß sektorale Mobilität nicht notwendigerweise von beruflicher Mobilität begleitet sein muß, da zahlreiche Berufe in verschiedenen Branchen eingesetzt werden können. Die Prozesse der beruflichen Mobilität sollten im Rahmen der vorliegenden Untersuchung ursprünglich ebenfalls erfaßt werden. Durch die oft ungenauen Antworten der Befragten (Berufsbezeichnung) wurde eine Auswertung diesbezüglicher Daten allerdings verunmöglicht.

8 In einer ähnlich gelagerten Untersuchung von FOPP (1975, S. 188) erweisen sich folgende Motive der Stellenwahl als besonders wichtig:

1. Bildungsgerechte Arbeit;

2. Aufstiegs- und Weiterbildungsmöglichkeiten;

3. Lohn- und Sozialleistungen;

4. Erreichbarkeit des Arbeitsplatzes;

5. Kontaktintensive Arbeit.

${ }^{9} \mathrm{Zu}$ gleichlautenden Ergebnissen kommen zum Beispiel LUTZSENGENBERGER (1974, S. 16).

10 FISCHER (1973, S. 68) stellt in diesem Zusammenhang präzisierend fest, daß das Aufstiegsmotiv am Anfang einer beruflichen Laufbahn noch relativ unbedeutend ist, aber nach dem zweiten oder dritten Stellenwechsel immer mehr in den Vordergrund tritt.

11 In diesem Sinne äußert sich etwa ABT (1980, S. 26).

12 Diese Befunde passen nahtlos ins Bild der bisherigen empirischen Wanderungsforschung. Vgl. z. B. ZIMMERMANN/ ANDERSECK/REDING/ZIMMERMANN (1973, S. 165); SCHUSTER (1969, S. 531); HAAG (1977, S. 27) oder HOFBAUER/NAGEL (1973, S. 259). 
13 Ähnliche Diagnosen der Probleme peripherer Arbeitsmärkte stellen z. B. auch DERENBACH (1982, S. 359) oder RECKER/SCHUETTE (1982, S. 554).

14 Dieser Befund ist insofern mit Vorsicht aufzunehmen, als bei schriftlichen Befragungen in der Regel ein schichtspezifischer Rücklauf zu erwarten ist und die Ausländer demzufolge häufig untervertreten sind.

15 Hinweise auf die diesbezüglichen relevanten Strategieüberlegungen findet man z. B. bei PRETAT (1972, S. 363 und 370 f.) oder bei STOCKER (1973, S. 85 f.).

16 Die geschlechtsspezifische Differenzierung zeigt, daß bei den Männern der Arbeitsplatz als dominierendes Wanderungsmotiv anzusehen ist. Bei den Frauen ist hingegen vor allem der Stellenwechsel des Ehepartners entscheidender Umzugsgrund. Diese Befunde belegen recht deutlich, daß das Wanderungsverhalten der Familie in erster Linie durch den Mann geprägt wird, und zwar auch in jenen Fällen, in denen die Frau ebenfalls erwerbstätig ist. Zu gleichlautenden Ergebnissen kommt z. B. ANDERSECK (1973).

17 Die mit der gleichen methodischen Grundlage (gleicher Katalog der Wohnortfaktoren) durchgeführten Untersuchungen von BUEHLER (1984, S. 88 f.) und KELLER (1983, S. 165 f.) kommen zu recht ähnlichen Ergebnissen. Unabhängig von der Region erscheint die Umweltqualität immer als herausragend wichtige Wohneigenschaft. Die medizinische und schulische Infrastruktur belegen ebenfalls in allen Bewertungsprofilen einen Spitzenplatz. Als von nachrangiger Bedeutung erweisen sich in sämtlichen Testregionen die kulturellen Freizeitangebote.

\section{Literaturverzeichnis}

ABT R. (1980): Praxisorientierte Potentialbestimmung für nicht-touristische Entwicklungen im Berggebiet. Arbeitsberichte des NFP "Regionalprobleme", Nr. 9, Bern.

ANDERSECK K. (1973): Der Einfluß der Ehefrau in seiner Bedeutung für das regionalpolitische Instrumentarium. Schriftenreihe der Gesellschaft für Regionale Strukturentwicklung, Bd. 2a, Bonn.

BASSAND M./BRULHARDT M. C. (1980): Mobilité spatiale. Publikationen des Schweizerischen Nationalfonds aus den Nationalen Forschungsprogrammen, Bd. 5, St-Saphorin.

BASSAND M./BRULHARDT M. C./HAINARD F./SCHULER M. (1985): Mobilité spatiale en Suisse, Lausanne.

BUEHLER-CONRADE. (1984): Bildung und regionale Entwicklung. Eine empirische Untersuchung über die regionalpolitische Bedeutung von Maßnahmen zur Verbesserung des Bildungsangebotes, dargestellt am Beispiel von Mittelschulen und Berufsschulen im schweizerischen Berggebiet, Bern/Frankfurt a. M./New York.

BUTTLER F./GERLACH K./LIEPMANN P. (1975): Funktionsfähige regionale Arbeitsmärkte als Bestandteil ausgewogener Funktionsräume. Veröffentlichungen der Akademie für Raumforschung und Landesplanung, Forschungs- und Sitzungsberichte, Bd. 94, Hannover.

DERENBACHR. (1982): Qualifikation und Innovation als Strategie der regionalen Entwicklung. In: Informationen zur Raumentwicklung, Heft 6/7, Bonn.
FISCHER G. (1973): Praxisorientierte Theorie der Regionalforschung, Tübingen.

FOPP L. (1975): Die Bedeutung des Branchen-Images für Stellenwahl und Stellenwechsel, Bern und Frankfurt.

FREUND U./ZABEL G. (1978): Regionale Wirkungen der Wirtschaftsstrukturförderung. Schriftenreihe "Raumordnung" des Bundesministers für Raumordnung, Bauwesen und Städtebau 06.023, Bonn.

GATZWEILER H. P. (1975): Zur Selektivität interregionaler Wanderungen, Forschungen zur Raumentwicklung, Bd. 1, Bonn.

HAAG F. (1977): Interregionale Wanderungen in der Schweiz. In: ORL-DISP, Nr. 44, Zürich.

HANSER CH. (1986): Erfolgskontrolle der Industrieansiedlungspolitik, Bern/Frankfurt a. M./New York.

HANSER CH. (1982): Auswirkungen von Massnahmen zur Erschließung von Industriezonen. In HANSER CH./HUBER S.: Hat die traditionelle Infrastrukturförderung für ländliche Regionen ausgedient? Themaband des NFP "Regionalprobleme», Diessenhofen.

HOFBAUER H./NAGEL R. (1973): Regionale Mobilität bei männlichen Enwerbspersonen in der Bundesrepublik Deutschland. In: Mitteilungen aus der Arbeitsmarkt- und Berufsforschung, Heft 3, Nürnberg.

KELLER T. (1983): Tourismus und Berggebietsförderung, Zürich.

LUTZ B./SENGENBERGER W. (1974): Arbeitsmarktstrukturen und öffentliche Arbeitsmarktpolitik, eine kritische Analyse von Zielen und Instrumenten. Schriftenreihe der Kommission für wirtschaftlichen und sozialen Wandel, Bd. 26, Göttingen.

NUPPNAU H. (1974): Wirkungen der Zonenrandförderung Analyse der regionalen Einkommenseffekte, Hamburg.

OECHSLIN R. (1981): Analyse regionaler Disparitäten, Aufbau eines Indikatorenkataloges zur Erfassung der regionalpolitischen Problemlage in der Schweiz, Zürich.

PRETAT CH. (1972): Anleitung zur Formulierung regionaler Entwicklungsziele. In: STOCKER PAUL (Präs.): Grundlagen zu den Leitlinien für die Berggebietsförderung, Bern.

RECKER E./SCHUETTE G. (1982): Räumliche Verteilung von qualifizierten Arbeitskräften und regionale Innovationstätigkeit. In: Informationen zur Raumentwicklung, Heft 6/7, Bonn.

SCHULER M. (1984): Migrationsentwicklung im schweizerischen Berggebiet. In: BRUGGER E. A./FURRER G./MESSERLI B./MESSERLIP. (Hrsg.): Umbruch im Berggebiet, Bern und Stuttgart

SCHUSTER L. (1969): Die Mobilität der Arbeitnehmer: Eine vergleichende Analyse von vier Erhebungen. In: Mitteilungen aus der Arbeitsmarkt- und Berufsforschung, Bd. 2, Nürnberg.

SPEHL H./TOEPFER K./TOEPFER P. (1979): Folgewirkungen von Industrieansiedlungen. Schriftenreihe der Gesellschaft für Regionale Strukturentwicklung, Bd. 3, Bonn.

STOCKER P. (Präs.) (1973): Leitlinien für die Berggebietsförderung, Bern.

ZIMMERMANN H./ANDERSECK K./REDING K./ZIMMERMANN A. (1973): Regionale Präferenzen, Wohnorientierung und Mobilitätsbereitschaft der Arbeitnehmer als Determinanten der Regionalpolitik. Schriftenreihe der Gesellschaft für Regionale Strukturentwicklung, Bd. 2, Bonn. 